Check for updates

Cite this: RSC Adv., 2018, 8, 9979

Received 22nd December 2017 Accepted 27th February 2018

DOI: $10.1039 / c 7 r a 13577 g$

rsc.li/rsc-advances

\section{Highly photoluminescent and stable silicon nanocrystals functionalized via microwave-assisted hydrosilylation $\uparrow$}

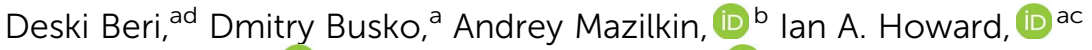 \\ Bryce S. Richards (D) *ac and Andrey Turshatov (D) *a
}

\begin{abstract}
Herein, we report a microwave-assisted hydrosilylation $(\mathrm{MWH})$ reaction for the surface passivation of silicon nanocrystals (Si-NCs) with linear alkenes. The MWH reaction requires only 20 minutes and allows us to produce Si-NCs with high photoluminescence quantum yields (PLQYs), reaching 39\% with an emission maximum of $860 \mathrm{~nm}$. Furthermore, we investigated the effect of ligand length on the photoluminescence properties of Si-NCs. We tested six alkenes with an even number of carbon atoms (from hexene-1 to hexadecene-1). The highest PLQY combined with a long stability (test period of 6 months) was observed when capping with the shortest ligand, hexene-1. The use of microwave heating turns the hydrosilylation step into a facile and sustainable process. In order to provide insight into the emissive properties of Si-NCs surface oxidation and luminescence decay were investigated using Fourier-transform infrared spectroscopy and time-resolved photoluminescence measurements.
\end{abstract}

\section{Introduction}

Silicon nanocrystals (Si-NCs) have attracted much research interest over the last few decades because of their potential applications in optoelectronics, photovoltaics, and photonics, as well as in the biomedical field for cancer treatment. ${ }^{1}$ Silicon (Si) is an extremely abundant and non-toxic element contained within the earth's crust. ${ }^{2}$ Bulk crystalline silicon (c-Si) exhibits an indirect band gap; in order to emit a photon a conduction-band electron needs phonon assistance to recombine with a valence-band hole. This means that photon emission in c-Si is slow, hence nonradiative recombination processes usually dominate and, as such, c-Si is a poor light emitter. However, when c-Si becomes finely divided onto a nanometer scale close to its free exciton Bohr radius ( $4.3 \mathrm{~nm}$ for bulk $\mathrm{c}-\mathrm{Si}$ ), its photophysics are altered due to the quantum confinement that takes place. In this regime, the electron no longer needs a phonon to recombine with the hole, so Si-NC are able to emit light in the red or near-infrared (NIR) region. Nevertheless, due to increased reactivity of Si-NCs towards

${ }^{a}$ Institute of Microstructure Technology, Karlsruhe Institute of Technology, Hermann-von-Helmholtz-Platz 1, 76344, Eggenstein-Leopoldshafen, Germany. E-mail: andrey.turshatov@kit.edu; bryce.richards@kit.edu

${ }^{b}$ Institute of Nanotechnology, Karlsruhe Institute of Technology, Hermann-vonHelmholtz-Platz 1, 76344 Eggenstein-Leopoldshafen, Germany

'Light Technology Institute, Karlsruhe Institute of Technology, Engesserstrasse 13, 76131 Karlsruhe, Germany

${ }^{d}$ Chemistry Department, Universitas Negeri Padang, Jl. Hamka, Air Tawar, 25131, Indonesia

$\dagger$ Electronic supplementary information (ESI) available: TEM images, luminescence spectra and decays and FTIR spectra. See DOI: 10.1039/c7ra13577g oxygen and water molecules, the synthesis of Si-NCs with high photoluminescence quantum yield (PLQY) is challenging.

There are two the promising methods to produce large quantities of luminescent Si-NCs, namely synthesis in a nonthermal plasma reactor or high temperature disproportionation reaction of silicon oxide based materials - silicon monoxide $\mathrm{SiO}_{x}$ or hydrogen silsesquioxanes $\left(\mathrm{HSiO}_{1.5}\right)_{n}-$ and subsequent functionalization of the hydrogen capped nanocrystals (H-Si-NCs) with organic ligands. ${ }^{3-12}$ The functionalization of Si-NCs relies on radical hydrosilylation reactions, initiated by temperature, light or radical initiators. The functionalization is necessary as it significantly increases the PLQY and chemical stability of the Si-NCs. The aforementioned method is able to provide suspensions of Si-NCs with maximum PLQY of $20-35 \%$ with emission in the NIR region. ${ }^{13-16}$

There are several reports with extraordinarily high PLQYs. For instance, Meinardi et al. reported PLQY of 50\% for NIR emission of Si-NCs synthesized in a non-thermal plasma reactor and capped with dodecene-1. ${ }^{\mathbf{1 7}}$ A long shelf-life of dodecyl-SiNCs with PLQY of $62 \%$ (the PLQY remained unchanged after two months under ambient conditions) was reported recently by Islam et al. ${ }^{18}$ Sangghaleh et al. reported PLQY of $60 \%$ for Si-NCs (capped with methyl undecanoate) prepared from silsesquioxanes. ${ }^{19}$ Qian et al. described that the stability of Si-NCs can be increased by using of fluorinated capping ligands. ${ }^{20}$ In addition, embedding Si-NCs into polymer matrix is very promising way to achieve exceptionally high PLQY (65\%) and long-term chemical stability. ${ }^{21}$ Even though high values of PLQY have been reported recently, but still, there is a lack of systematic study of the longterm stability of Si-NCs (shelf-life under ambient conditions). 
In our work, we annealed silicon monoxide $\mathrm{SiO}_{x}$ in reducing atmosphere and elaborated the hydrogen terminated Si-NCs via reaction with hydrofluoric acid. We optimized hydroxylation step realized using microwave-assisted hydrosilylation (MWH) and addressed the investigation of photoluminescence and stability of Si-NCs obtained via the MWH step. MWH is an advanced process of the Si-NCs functionalisation which is able to reduce reaction time, improving the product quality, product reproducibility and a cleaner synthesis. ${ }^{\mathbf{4 , 2 2}}$ In our report we demonstrate that at higher temperatures, $\mathrm{MWH}$ can run 10-30 times faster than conventional heating and the reaction can yield Si-NCs with high PLQY (up to 35\%) and excellent shelf-life (6 months). In addition, we investigated the effect of using alkenes with different length ( $N$-ene-1, where $N$ is number of carbon atoms in the linear alkyl chain) on the photophysical properties of Si-NCs as well as the shelf-life stability of the product.

\section{Results and discussion}

In order to elucidate the effect of the organic ligands on the luminescent properties of the Si-NCs all chemical steps preceding the hydrosilylation reaction were performed under similar conditions. Reduction of $\mathrm{SiO}_{x}$ in continuous flow of $\mathrm{Ar} /$ $\mathrm{H}_{2}$ gas and subsequent etching with $48 \% \mathrm{HF}$ solution leads to the formation of hydrogen-capped Si-NCs. Subsequently, within 20 minutes these are converted in a microwave reactor to form stable Si-NCs capped with organic ligands. We measured the PLQY of the freshly prepared samples. Afterwards, each sample was divided into two vials. One vial was placed in a glovebox with inert atmosphere and used as a control experiment. The second part of the suspension was stored in the dark in closed vials. The vials were opened regularly for PLQY measurements. Thus, no special precautions were attempt for the storage of the Si-NCs placed in the second vial.

The data of Fig. 1a and b reveals diamond cubic crystal structure of synthesized Si-NCs. High resolution transmission

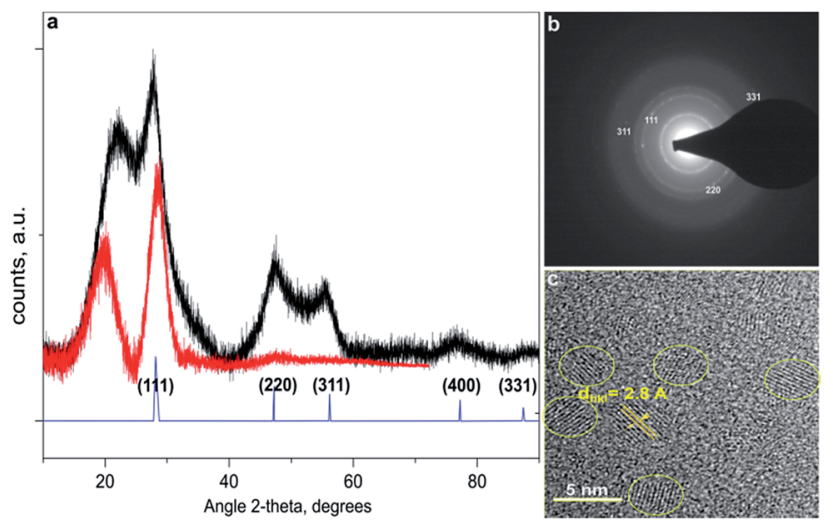

Fig. 1 (a) Representative X-ray diffractograms of $\mathrm{Si}-\mathrm{NCs}$ in $\mathrm{SiO}_{x}$ matrix before HF etching (black line) and free standing Si-NCs capped with decene-1 along with JCPDS 027-1402; (b) electron diffraction data for free standing Si-NCs capped with decene-1; (c) HRTEM image of free standing Si-NCs capped with decene-1. electron microscopy (HRTEM) (Fig. 1c) confirms the crystal structure of Si-NCs with interplanar distance of $0.32 \mathrm{~nm}$ corresponding to the distance (111) in c-Si. The average size of Si-NCs realized in this work is $\sim 4.6 \pm 0.3 \mathrm{~nm}$ (sum of six independent etching processes), as deduced from transmission electron microscopy (TEM) images (see Fig. S1 $\dagger$ ). Importantly, TEM results indicate that the average size of the Si-NCs core remains the same after the surface passivation step with all investigated ligands (Fig. 2 and $\mathrm{S} 1 \dagger$ ). In contrast, the results of DLS measurements (Fig. 2) indicate an increase of the hydrodynamic diameter in case of surface passivation with dodecene-1 and longer hydrocarbons. Previously, Yang et al. ${ }^{23}$ reported the existence of a polymer corona forming around the Si-NCs core during the hydrosilylation process. They found that oxygen, high ligand concentration, and increase of temperature promote chain propagation in oligomerization reactions and lead to formation of an organic corona. In our experiment, we observed that the size and the hydrodynamic diameter of the nanocrystals are consistent in case of capping with relatively short ligands, e.g. hexene-1, octene- 1 and decene- 1 , whereas the use of the long ligands (with $N \geq 12$ ) leads to of the growth of a thick organic shell surrounding the silicon core and a discrepancy between the particle size measured by TEM (seeing only the Si-NCs core) and DLS (seeing the Si-NCs core plus organic corona).

Fig. 3 displays the photoluminescence (PL) spectra of the synthesized Si-NCs. Under ultraviolet (UV, $375 \mathrm{~nm}$ ) excitation all nanocrystals demonstrate strong emission in the NIR part of the electromagnetic spectrum. The effective mass approximation (EMA) ${ }^{24,25}$ model predicts (eqn $(1)^{26}$ ) that uniform Si-NCs with size $4.6 \mathrm{~nm}$ emit photons with energy $E_{\mathrm{PL}}=1.55 \mathrm{eV}$ $\left(\lambda_{\max }=800 \mathrm{~nm}\right)$.

$$
E_{\mathrm{PL}}=E_{0}+\frac{\hbar^{2} \pi^{2}}{2 R^{2}}\left(\frac{1}{m_{\mathrm{e}}^{*}}+\frac{1}{m_{\mathrm{h}}^{*}}\right)-\frac{1.786 e^{2}}{\varepsilon_{\mathrm{r}} R}
$$

where $E_{0}$ is band gap of c-Si, h is Planck constant with stroke, $R$ radius of Si-NCs, $m_{\mathrm{e}}^{*}=0.19 m_{0}$ and $m_{\mathrm{h}}^{*}=0.286 m_{0}$ are effective masses of electrons and holes in Si-NCs correspondingly, $m_{0}-$ electron mass, $e$ - charge of electron, $\varepsilon_{\mathrm{r}}$ - relative permittivity of Si-NCs.

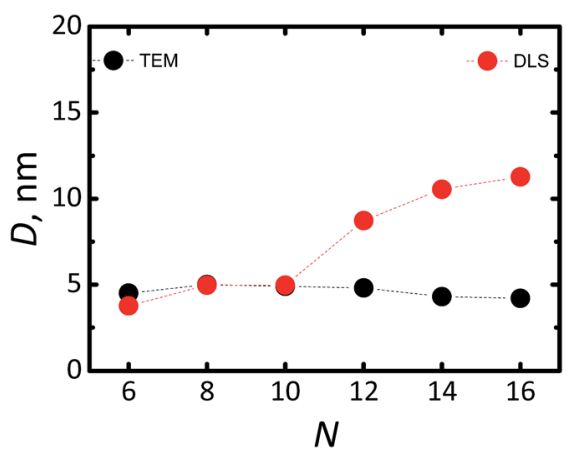

Fig. 2 Average diameter of Si-NCs estimated with TEM (black cycles) and DLS (red cycles) as function of number $(N)$ of carbon atoms in the linear aliphatic chain of the capping ligand. 


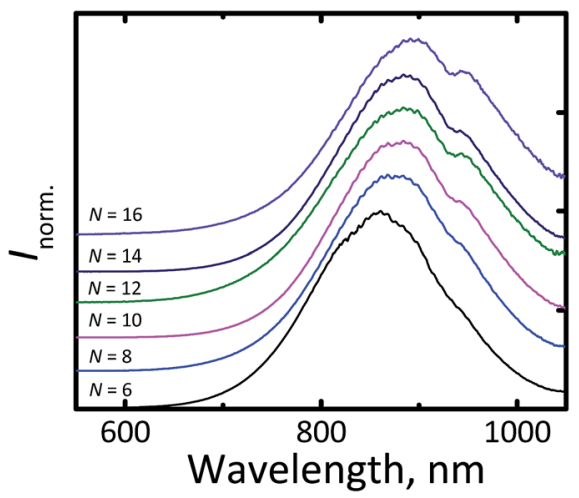

Fig. 3 Normalized fluorescent spectra of Si-NCs with different capping ligands. $N$ is the number of carbon atoms in the linear aliphatic chain of the capping ligand.

In our measurements, we observed an unusually large red shift in the position of the luminescence maximum $\left(\lambda_{\max }=\right.$ 850-905 nm) for all ligands, as shown in Fig. 3 (it should be noted that the local minimum at $920 \mathrm{~nm}$ can be explained by absorption of the solvent associated with the overtone of $\mathrm{C}-\mathrm{H}$ stretching vibration). This effect cannot be explained by a special condition (MW heating) of the hydrosilylation process.

The same shift in the position of the emission maximum was observed for Si-NCs prepared by hydrosilylation via conventional heating in agreement with experimental procedure described by Sun et al. ${ }^{6}$ (see Fig. S2 $\dagger$ ). We assume that the shift can be explained by a polydispersity of the Si-NCs ensembles. A broad sized distribution results the red shift in a position of an emission maximum in comparison with the theoretical prediction for monodisperse $\mathrm{Si}-\mathrm{NCs}$, as it has been recently described by $\mathrm{Yu}$ et al. ${ }^{15}$ Fig. S3† supports this statement: we observed the clear correlation between FWHM of the emission peak and position of the luminescence maximum. The broader emission peaks is the more red shifted peaks are observed.

In order to quantify luminescence of Si-NCs we measured absolute PLQY for all samples. The results of the PLQY measurements are summarized in Fig. 4.

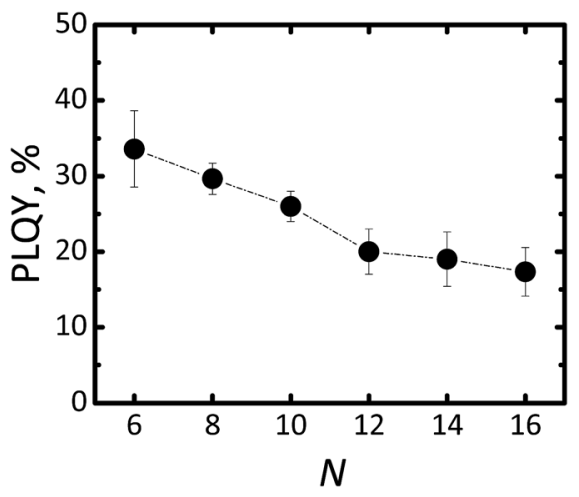

Fig. 4 Changing of PLQY for Si-NCs functionalised with different capping ligands. $N$ is the number of carbon atoms in the linear aliphatic chain of the capping ligand.
The highest PLQY value of $34 \pm 4 \%$ was observed for capping with the shortest ligand - hexene-1. Importantly, the error bar of PLQY values was derived from three independent experiments for each capping ligand, except for hexene-1 for which six independent experiments were performed. The general trend indicates that the longer the ligand is the lower the PLQY.

The lowest PLQY of $18 \pm 2 \%$ was obtained for hexadecene- 1 . Our first hypothesis was that the partial surface oxidation of the Si-NCs is responsible for decreasing the PLQY in the case of ligands with longer carbon chains. This hypothesis might be supported by an analysis of FTIR spectra of capped Si-NCs. Fig. 5a displays a selected region of the FTIR spectra measured for Si-NCs capped with different ligands. We attribute the peak at $800 \mathrm{~cm}^{-1}$ to $\mathrm{Si}-\mathrm{C}_{\text {(stretching) }}$ vibration, whereas the broad peak at 960-1140 $\mathrm{cm}^{-1}$ to $\mathrm{Si}-\mathrm{O}_{(\text {stretching) }}$ vibration. The $\mathrm{Si}-\mathrm{C}_{(\text {stretching) }}$ vibration can overlap with $\mathrm{Si}-\mathrm{O}_{(\text {bending) }}$ peak (observed at 797$\left.810 \mathrm{~cm}^{-1}\right)$. However the $\mathrm{Si}-\mathrm{O}_{(\text {bending) }}$ peak has typically much low intensity then $\mathrm{Si}-\mathrm{C}_{(\text {stretching) }}$ and, thus, has minor impact in the further calculations. We did not observed the characteristic peak of $\mathrm{Si}-\mathrm{H}$ vibration at $900 \mathrm{~cm}^{-1}$ that indicates about complete substitution of $\mathrm{Si}-\mathrm{H}$ bonds due to oxidation and hydrosilylation. We expect that the ratio in the eqn (2) can provide qualitative information about surface oxidation of Si-NCs. We normalized the results in the way, that the integral $A_{\bar{\nu}(\mathrm{Si}-\mathrm{C})}=1$, so the value of the integral $A_{\bar{\nu}(\mathrm{Si}-\mathrm{O})}$ reflects relative amount of oxidation occurring at the Si-NCs surface. The large $A_{\bar{\nu}(\mathrm{Si}-\mathrm{O})}$ is the stronger oxidation occurs at the surface.

$$
\frac{A_{\bar{\nu}(\mathrm{Si}-\mathrm{C})}}{A_{\bar{\nu}(\mathrm{Si}-\mathrm{O})}}=\frac{\int_{760 \mathrm{~cm}^{-1}}^{830 \mathrm{~cm}^{-1}} \bar{v}_{\left(760-830 \mathrm{~cm}^{-1}\right)} \mathrm{d} \bar{v}_{\left(760-830 \mathrm{~cm}^{-1}\right)}}{\int_{960}^{1140} \bar{v}_{\left(960-1140 \mathrm{~cm}^{-1}\right)} \mathrm{d} \bar{v}_{\left(960-1140 \mathrm{~cm}^{-1}\right)}}
$$
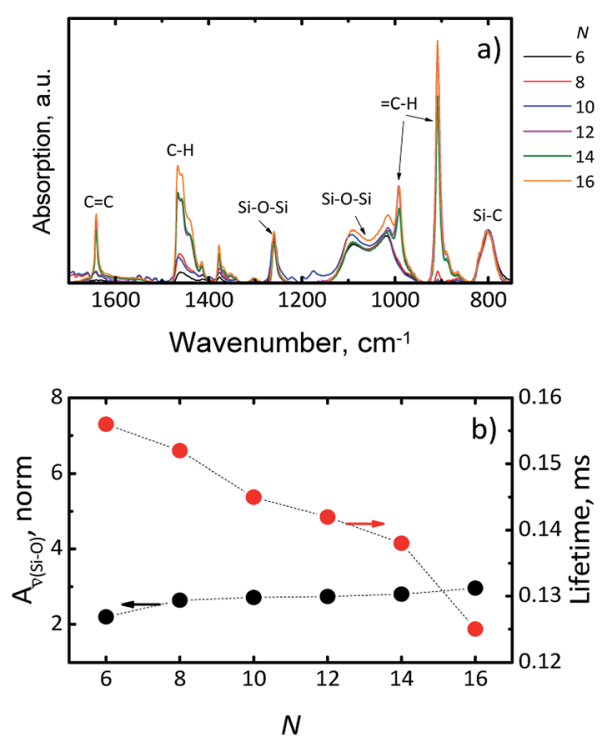

Fig. 5 (a) Attenuated total reflectance infrared (ATR-FTIR) spectrum of Si-NCs with different capping ligands. $N$ is the number of carbon atoms in the linear aliphatic chain of the capping ligand. (b) $A_{\bar{\nu}(\mathrm{Si}-\mathrm{O}) \text { and }}$ luminescence lifetime as function of number $(N)$ of carbon atoms in the capping ligand. 
Fig. 5b indicates only the small increase of $A_{\bar{\nu}(\mathrm{Si}-\mathrm{O})}$ with the increase of the length of hydrocarbon chain. Thus, the surface oxidation is apparently not the key phenomenon responsible for the PLQY decrease. Surprisingly, washing several times the SiNCs with hexane doesn't eliminates dodecene-1, tetradecene-1 and hexadecene- 1 from Si-NCs. FTIR spectrums of Si-NCs capped with the long chain ligands display additional peaks corresponding to the $-\mathrm{C}=\mathrm{C}-$ and $=\mathrm{C}-\mathrm{H}$ bonds. The FTIR spectrums (Fig. 5a) also display a band at $1259 \mathrm{~cm}^{-1}$ that can be attributed to longitudinal phonons relating to $\mathrm{Si}-\mathrm{O}-\mathrm{Si}$ bond with the deformed bond angle $\sim 142^{\circ} .^{27}$ This peak is well separated from the other $\mathrm{Si}-\mathrm{O}-\mathrm{Si}$ peak at $960-1140 \mathrm{~cm}^{-1}$ and its intensity does not change for different capping ligands. Though the existence such preferential configuration of $\mathrm{Si}-\mathrm{O}-\mathrm{Si}$ bond is unclear, we assume that this peak represents oxidation sites of Si-NCs surface originating from nature of $\mathrm{SiO}_{x}$ material because the similar peak is not observed in Si-NCs prepared from silsesquioxanes. ${ }^{18,26,28,29}$

An alternative explanation for the changes in PLQY with length of the capping ligands can be proposed using work of Aharoni et $a l^{30}$ They described long-range electronic-tovibrational energy transfer from excited quantum dots to matrix vibrational overtones. Fig. $\mathrm{S} 4 \dagger$ depicts emission spectrums of Si-NCs capped with hexene-1 and hexadecene-1 and absorption spectrums of solvents (hexene- 1 and hexadecene-1). The absorption spectrums of the alkenes (similar for all investigated alkenes) demonstrate $\mathrm{C}-\mathrm{H}$ vibrational overtones with the absorption maximums peaking at 923-930 $\mathrm{nm}$ that perfectly match emission spectrums of Si-NCs. The matching is better for Si-NCs with long capping ligands. Thus, both solvent and capping ligands (forming the polymer corona) can act as an efficient quencher of excited Si-NCs. Indeed, Fig. 5b displays a decrease of the radiative lifetime in range $N=6 \rightarrow N=16$ from $0.156 \mathrm{~ms}$ to $0.125 \mathrm{~ms}$ (luminescence decays are presented in Fig. S5 $\dagger$ ). The decrease of the lifetime signalizes about increase of non-radiative deactivation and is in good agreement with observed decrease of PLQY.

The presence of oxidized surface sites related to $\mathrm{Si}-\mathrm{O}-\mathrm{Si}$ bonds (from FTIR data) can also reduce PLQY of Si-NCs. We observed oxidation independently from used alkenes and methods of deoxygenation to prevent the oxidation. Apparently, these type of surface defects limits the highest achievable PLQY to $39 \%$ - or an average value of $34 \pm 5 \%$ calculated from six independent syntheses - achievable by the method used in the current work.

To complete the characterization of the Si-NCs we systematically investigated the shelf-life of the synthesized materials. Fig. 6 displays PLQY values taken during the period six months. We did not observe significant altering of photoluminescence emission after six months of the storage in dark ambient conditions (PLQY/PLQY $>0.9$ ). The part of the samples stored in a glovebox displays PLQY comparable with PLQY of samples stored under ambient conditions. FTIR spectrums of samples stored in a glovebox indicate also unchanged ratio between $\mathrm{Si}-\mathrm{C}$ and $\mathrm{Si}-\mathrm{O}$ bonds. These experimental observations confirm high stability of Si-NCs functionalized via microwave-assisted hydrosilylation.

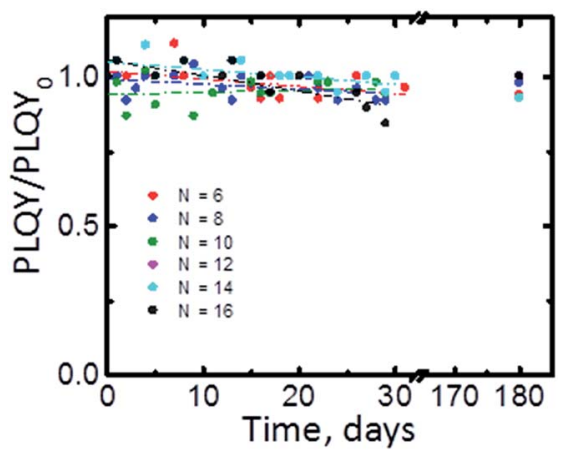

Fig. 6 PLQY of Si-NCs stored in ambient as function shelf-life time for Si-NCs with different capping ligands. $N$ is the number of carbon atoms in the linear aliphatic chain of the capping ligand.

\section{Experimental}

\section{Materials}

Silicon monoxide (99.9\%, 325 mesh) was purchased from Sigma-Aldrich, hydrofluoric acid (48\%) was purchased from Fisher Scientific, ethanol absolute, methanol HPLC grade, toluene (99\%), dodecene-1 (94\%), tetradecene-1 (96\%), hexadecene-1 (98\%), and octadecene-1 (96\%) were purchased from Merck, hexene-1 (99\%) and octane-1 (99\%) were purchased from Acros, decene-1 (96\%) and $n$-hexane spectroscopic grade were purchased from Alfa-Aesar.

\section{Synthetic procedures}

Synthesis of hydrogen-terminated Si-NCs. $1.0 \mathrm{~g} \mathrm{SiO}_{n}$ was transferred to a quartz boat and reduced thermally under flowing $\mathrm{H}_{2}(5 \%)$ in $\mathrm{Ar}(95 \%)$ at $900{ }^{\circ} \mathrm{C}$ for 60 minutes in a quartz tube furnace. After annealing, the product was cooled down to room temperature and grounded using agate mortar. The fine powder was transferred to $100 \mathrm{ml}$ Teflon vessel and $20 \mathrm{ml}$ of absolute ethanol and $20 \mathrm{ml}$ of $\mathrm{HF}$ acid solution $48 \% \mathrm{v} / \mathrm{v}$ were added and stirred magnetically for 150 minutes to remove the $\mathrm{SiO}_{x}$ matrix. The brown dispersion was transferred to a Teflon separatory funnel and extracted with $20 \mathrm{ml}$ of corresponding alkene. The yellowish Si-NCs dispersion was discarded and transferred a microwave glass tube. Argon gas was purged into the dispersion for 20 minutes before reaction in a microwave (MW) reactor.

Hydrosilylation using MW reactor. The G30 tube was placed into MW chamber (Anton Paar Monowave 400) and heated thermally at $250{ }^{\circ} \mathrm{C}$ for 20 minutes in $600 \mathrm{rpm}$ stirring. The temperature of the solution was monitored a ruby thermometer dipped inside the solution. After reaction complete, product was transferred to centrifuge tube and centrifuged at $4500 \mathrm{rpm}$ for $10 \mathrm{~min}$ to separate large particles.

\section{Characterization}

A UV-vis-NIR spectrophotometer (Perkin Elmer Lambda 950) was used to measure optical absorption of materials. A spectrofluorometer (Varian Cary Eclipse) was used for measuring excitation spectra. A FT-IR spectrometer (Bruker Vertex 70 with 
platinum ATR module) was used for measuring IR absorption. Dynamic light scattering (Anton Paar Litesizer 500) was used for measuring the size of particles dispersed in a liquid. PL emission spectra were measured with calibrated CCS200 spectrometer (Thorlabs) using $1 \mathrm{~mW}$ LED with $\lambda=375 \mathrm{~nm}$ for excitation. Absolute PLQY was measured using de Mello methods. ${ }^{31}$ The LED beam was focused by a lens and directed into an integrating sphere (Labsphere) with a diameter of $15 \mathrm{~cm}$. An optical fiber with a diameter of $1 \mathrm{~mm}$ (FP1000URT, Thorlabs) was used for collection of the emission from the integrating sphere and transferring this to the spectrometer (CCS200, Thorlabs). During the absorption measurement (measurement of the LED at the direct and indirect excitation of the sample and empty sphere), short integration times, usually 20-50 times shorter than for UC detection, were utilized. All raw detected spectra were recalculated to give power spectra using an integration time value. The linearity of the signal versus integration time of CCD was proven experimentally. The spectral response of the whole detection system was calibrated using a calibration lamp (HL-3plus-INT-CAL, Ocean Optics) and the correction was further applied to the power spectra. For the PL lifetime measurements, time-correlated single photon counting (TCSPC) and a multichannel scaling (MCS) card (Timeharp 260, PicoQuant) were used. The modulation of the diode laser (Thorlabs $405 \mathrm{~nm}$ ) was performed via a built-in function generator in the laser diode driver. In order to detect rise and fall times of the UC emission, the TTL signal from the laser diode controller was delayed by the use of a delay generator (DG645, Stanford Research Systems). The spectral separation of the photoluminescence was achieved via a double monochromator (DTMS300, Bentham) and the emission at specific wavelength was detected via a photomultiplier tune (R928P, Hamamatsu), mounted in temperature-cooled housing (CoolOne, Horiba).

TEM investigations were carried out on a TITAN 60-300 transmission electron microscope at accelerating voltage $300 \mathrm{kV}$.

\section{Conclusions}

To conclude, MWH in very short step of 20 minutes allows getting Si-NCs with high PLQY of $39 \%$ and emission maximum of $860 \mathrm{~nm}$. The highest PLQY is achieved through functionalisation with hexene-1 - the shortest ligand among investigated alkenes. We attribute increase of PLQY in case of capping with hexene-1 with reduce of long-range electronic-to-vibrational energy transfer from quantum dots to matrix vibrational overtones. Furthermore, Si-NCs prepared from silicon monoxide $\mathrm{SiO}_{n}$ bear sufficient number of surface defects associated with deformed Si-O-Si bonds, which can intrinsically limit the values of PLQY prepared form this type of precursor. Meanwhile, MWH results highly luminescent Si-NCs with long shelflife at the ambient conditions.

\section{Conflicts of interest}

There are no conflicts to declare.

\section{Acknowledgements}

The authors would like also to acknowledge the financial support provided by the Helmholtz Association: (i) a Recruitment Initiative Fellowship for BSR; (ii) the funding of chemical synthesis equipment from the Helmholtz Materials Energy Foundry (HEMF); (iii) Karlsruhe Nano Micro Facility (KNMF) and (iv) the Science and Technology of Nanosystems research programme (STN). DB acknowledges the Directorate General of Higher Education (DGHE) of the Republic of Indonesia for the PhD fellowship 101.2/E4.4/K/2015.

\section{Notes and references}

1 M. Dasog, J. Kehrle, B. Rieger and J. G. C. Veinot, Angew. Chem., Int. Ed., 2016, 55, 2322-2339.

2 A. Das and P. T. Snee, ChemPhysChem, 2016, 17, 598-617.

3 C. M. Hessel, D. Reid, M. G. Panthani, M. R. Rasch, B. W. Goodfellow, J. Wei, H. Fujii, V. Akhavan and B. A. Korgel, Chem. Mater., 2012, 24, 393-401.

4 J. Wang, Y. Liu, F. Peng, C. Chen, Y. He, H. Ma, L. Cao and S. Sun, Small, 2012, 8, 2430-2435.

5 M. Dasog, G. B. De los Reyes, L. V. Titova, F. A. Hegmann and J. G. C. Veinot, ACS Nano, 2014, 8, 9636-9648.

6 W. Sun, C. Qian, L. Wang, M. Wei, M. L. Mastronardi, G. Casillas, J. Breu and G. A. Ozin, Adv. Mater., 2015, 27, 746-749.

7 J. J. Wu and U. R. Kortshagen, RSC Adv., 2015, 5, 103822103828.

8 Z. Yang, C. M. Gonzalez, T. K. Purkait, M. Iqbal, A. Meldrum and J. G. C. Veinot, Langmuir, 2015, 31, 10540-10548.

9 Y. Yu and B. A. Korgel, Langmuir, 2015, 31, 6532-6537.

10 Y. Yu, C. E. Rowland, R. D. Schaller and B. A. Korgel, Langmuir, 2015, 31, 6886-6893.

11 M. Miyano, Y. Kitagawa, S. Wada, A. Kawashima, A. Nakajima, T. Nakanishi, J. Ishioka, T. Shibayama, S. Watanabe and Y. Hasegawa, Photochem. Photobiol. Sci., 2016, 15, 99-104.

12 T. K. Purkait, M. Iqbal, M. A. Islam, M. H. Mobarok, C. M. Gonzalez, L. Hadidi and J. G. C. Veinot, J. Am. Chem. Soc., 2016, 138, 7114-7120.

13 X. Liu, Y. Zhang, T. Yu, X. Qiao, R. Gresback, X. Pi and D. Yang, Part. Part. Syst. Charact., 2016, 33, 44-52.

14 A. Marinins, Z. Yang, H. Chen, J. Linnros, J. G. C. Veinot, S. Popov and I. Sychugov, ACS Photonics, 2016, 3, 1575-1580. 15 Y. Yu, G. Fan, A. Fermi, R. Mazzaro, V. Morandi, P. Ceroni, D.-M. Smilgies and B. A. Korgel, J. Phys. Chem. C, 2017, 121, 23240-23248.

16 M. L. Mastronardi, F. Maier-Flaig, D. Faulkner, E. J. Henderson, C. Kübel, U. Lemmer and G. A. Ozin, Nano Lett., 2012, 12, 337-342.

17 F. Meinardi, S. Ehrenberg, L. Dhamo, F. Carulli, M. Mauri, F. Bruni, R. Simonutti, U. Kortshagen and S. Brovelli, Nat. Photonics, 2017, 11, 177-185.

18 M. A. Islam, M. H. Mobarok, R. Sinelnikov, T. K. Purkait and J. G. C. Veinot, Langmuir, 2017, 33, 8766-8773. 
19 F. Sangghaleh, I. Sychugov, Z. Yang, J. G. C. Veinot and J. Linnros, ACS Nano, 2015, 9, 7097-7104.

20 C. Qian, W. Sun, L. Wang, C. Chen, K. Liao, W. Wang, J. Jia, B. D. Hatton, G. Casillas, M. Kurylowicz, C. M. Yip, M. L. Mastronardi and G. A. Ozin, J. Am. Chem. Soc., 2014, 136, 15849-15852.

21 A. Marinins, R. Zandi Shafagh, W. van der Wijngaart, T. Haraldsson, J. Linnros, J. G. C. Veinot, S. Popov and I. Sychugov, ACS Appl. Mater. Interfaces, 2017, 9, 3026730272.

22 W. Sun, C. Qian, M. L. Mastronardi, M. Wei and G. A. Ozin, Chem. Commun., 2013, 49, 11361-11363.

23 Z. Yang, M. Iqbal, A. R. Dobbie and J. G. C. Veinot, J. Am. Chem. Soc., 2013, 135, 17595-17601.

24 L. E. Brus, J. Chem. Phys., 1984, 80, 4403-4409.

25 L. E. Brus, J. Chem. Phys., 1983, 79, 5566-5571.
26 Z. Yang, G. B. De los Reyes, L. V. Titova, I. Sychugov, M. Dasog, J. Linnros, F. A. Hegmann and J. G. C. Veinot, ACS Photonics, 2015, 2, 595-605.

27 I. P. Lisovskii, V. G. Litovchenko, V. G. Lozinskii and G. I. Steblovskii, Thin Solid Films, 1992, 213, 164-169.

28 J. Kehrle, S. Kaiser, T. K. Purkait, M. Winnacker, T. Helbich, S. Vagin, J. G. C. Veinot and B. Rieger, Nanoscale, 2017, 9, 8489-8495.

29 R. J. Clark, M. Aghajamali, C. M. Gonzalez, L. Hadidi, M. A. Islam, M. Javadi, M. H. Mobarok, T. K. Purkait, C. J. T. Robidillo, R. Sinelnikov, A. N. Thiessen, J. Washington, H. Yu and J. G. C. Veinot, Chem. Mater., 2017, 29, 80-89.

30 A. Aharoni, D. Oron, U. Banin, E. Rabani and J. Jortner, Phys. Rev. Lett., 2008, 100, 057404.

31 J. C. de Mello, H. F. Wittmann and R. H. Friend, Adv. Mater., 1997, 9, 230-232. 